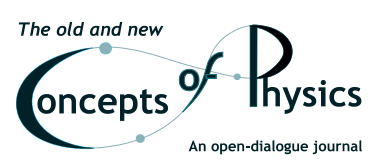

www.uni.lodz.pl/concepts

\title{
SQUEEZED AND ENTANGLED STATES OF A SINGLE SPIN
}

\author{
Barış Öztop, Alexander A. Klyachko and Alexander S. Shumovsky \\ Faculty of Science, Bilkent University \\ Bilkent, Ankara, 06800 Turkey \\ e-mail: shumo@fen.bilkent.edu.tr
}

(Received 23 December 2007; accepted 1 March 2007)

\begin{abstract}
We show correspondence between the notions of spin squeezing and spin entanglement. We propose a new measure of spin squeezing. We consider a number of physical examples.
\end{abstract}




\section{Barıș Öztop, Alexander A. Klyachko and Alexander S. Shumovsky}

It is well known that the concept of squeezed states [1] was originated from the famous work by N.N. Bogoliubov [2] on the superfluidity of liquid $\mathrm{He}^{4}$ and canonical transformations. Initially, it was developed for the Bose-fields. Later on, it has been extended on spin systems as well.

The two main objectives of the present paper are on the one hand to show that the single spin $s \geq 1$ can be prepared in a squeezed state and on the other hand to demonstrate one-to-one correspondence between the notions of spin squeezing and entanglement. The results are illustrated by physical examples.

Spin-coherent states - Historically, the notion of spin-coherent states had been introduced [3] before the notion of spin-squeezed states. In a sense, it just reflected the idea of Glauber [4] about creation of Bose-field coherent states from the vacuum by means of the displacement operator

$$
|\alpha\rangle_{\text {field }}=D(\alpha)|\mathrm{vac}\rangle, \quad D(\alpha)=\exp \left(\alpha a^{+}-\alpha^{*} a\right),
$$

where $\alpha \in \mathbb{C}$ is an arbitrary complex parameter and $a^{+}, a$ are the Boson creation and annihilation operators.

In the case of spin- $s$ system specified by the three spin operators $S_{x}, S_{y}, S_{z}$ that obey the commutation relations

$$
\left[S_{j}, S_{k}\right]=i \epsilon_{j k \ell} S_{\ell},
$$

the lowest spin state $|-s\rangle\left(S_{z}|-s\rangle=-s|-s\rangle\right)$ plays the role of the vacuum state, so that the spin-coherent state has the form

$$
|\alpha\rangle=\exp \left(\alpha S_{+}-\alpha^{*} S_{-}\right)|-s\rangle
$$

where

$$
S_{ \pm}=\frac{1}{\sqrt{2}}\left(S_{x} \pm i S_{y}\right)
$$

are the spin-ladder operators (compare with Eq. (1)). For extension of the notion of coherence on arbitrary Lie groups we refer to [5].

Since the Hermitian spin operators $S_{x}, S_{y}, S_{z}$ form an orthogonal basis of the $\mathfrak{s u}(2)$ algebra, the spin-displacement operator (exponential in Eq. (3) belongs to the $\mathrm{SU}(2)$ group, so that the spin-coherent 
state (3) can be considered as the $\mathrm{SU}(2)$ rotation of the minimal state $|-s\rangle$. In other words, there is a direction $\vec{e}_{\alpha}$ in the three-dimensional spin space (new quantization axis) such that the operator $S_{z, \vec{e}_{\alpha}}(z-$ component of the spin vector, which is now directed along the $\vec{e}_{\alpha}$ axis, has the same eigenvalue $-s$ in the state $|\alpha\rangle$.

As well as conventional coherent states, the spin-coherent states (3) can be specified by uncertainties of spin operators measured in those states. In view of the commutation relation (2), the Heisenberg uncertainty relation in an arbitrary state $\psi$ has the form

$$
V\left(S_{j}, \psi\right) V\left(S_{k}, \psi\right) \geq \frac{1}{4}\left|\left\langle\psi\left|S_{\ell}\right| \psi\right\rangle\right|^{2} .
$$

where

$$
V(X, \psi) \equiv\left\langle\psi\left|X^{2}\right| \psi\right\rangle-\langle\psi|X| \psi\rangle^{2}
$$

denotes the variance (uncertainty). Thus, in the coherent state (3), the uncertainty of the corresponding $z$-component $V\left(S_{z, \vec{e}_{\alpha}}, \alpha\right)=0$ and $\left|\left\langle\alpha\left|S_{z, \vec{e}_{\alpha}}\right| \alpha\right\rangle\right|=s$. In turn, the variances of the two spin operators orthogonal to $S_{z, \vec{e}_{\alpha}}$ are both equal to $s / 2$.

An important characteristics of states is provided by the total variance (see Ref. [6] and references therein)

$$
\mathbb{V}(\psi)=\sum_{j=x, y, z} V\left(S_{j}, \psi\right) .
$$

By definition, this quantity is invariant under rotations of the basis of the $\mathfrak{s u}(2)$ algebra. Thus, for an arbitrary spin-coherent state $\mathbb{V}(\alpha)=$ $s$. It can be easily seen that

$$
s \leq \mathbb{V}(\psi) \leq s(s+1),
$$

so that the spin-coherent state corresponds to the minimal total variance. It is also easily seen that any state of spin- $1 / 2$ system is a spin-coherent one.

Spin-squeezed states - In the case of Bose-fields, the simplest squeezed state is defined by action of the squeezing operator $\mathcal{S}(\zeta)$ on the vacuum state [1]

$$
|\zeta\rangle_{\text {field }}=\exp \left(\frac{1}{2} \zeta^{*} a^{2}-\frac{1}{2} \zeta a^{+2}\right)|\mathrm{vac}\rangle
$$




\section{Barıș Öztop, Alexander A. Klyachko and Alexander S. Shumovsky}

(for recent review, see [7]). For the two field quadratures $Q=\left(a^{+}+a\right)$ and $P=-i\left(a-a^{+}\right)$that obey the uncertainty relation

$$
V(Q, \psi) V(P, \psi) \geq 1,
$$

in the state (8) we have

$$
V(Q, \zeta)=e^{-2|\zeta|}, \quad V(P, \zeta)=e^{2|\zeta|},
$$

so that uncertainty of one of the quadratures can be squeezed with simultaneous enlargement of uncertainty of the other quadrature under the condition that $V(Q, \zeta) V(P, \zeta)=1$.

The attempts to define spin-squeezed states in direct analogy with the Bose-field squeezed states have failed (see critical review in Ref. [8]). An approach has been proposed in Ref. [8] considers the meanspin vector $\bar{S}_{\psi}$ with components $\left\langle\psi\left|S_{j}\right| \psi\right\rangle(j=x, y, z)$. Its direction again is chosen to be the $z$-axis. A spin state is called to be squeezed whenever the uncertainty in one of the spin components orthogonal to this direction is smaller than $s / 2$. For the spin-squeezed state, the inequality (7) takes the form

$$
\mathbb{V}_{\text {coh }}=s<\mathbb{V}(\zeta) \leq s(s+1)=\mathbb{V}_{\max } .
$$

Measure of spin squeezing - With the inequality (9) in hands, we can define a measure of squeezing of an arbitrary state of spin 1 . It is possible to carry the interval of this inequality into $[0,1]$ such as

$$
0<\frac{\mathbb{V}(\psi)-\mathbb{V}_{\text {coh }}}{\mathbb{V}_{\max }-\mathbb{V}_{\text {coh }}} \leq 1
$$

Thus, the measure $\nu(\psi)$ of squeezing becomes

$$
\nu(\psi)=\frac{\mathbb{V}(\psi)-\mathbb{V}_{\text {coh }}}{\mathbb{V}_{\max }-\mathbb{V}_{\text {coh }}},
$$

which takes the value 0 for spin-coherent states (no squeezing) and 1 for the maximally squeezed state.

Taking into account that

$$
\sum_{j=x, y, z}\left\langle\psi\left|S_{j}^{2}\right| \psi\right\rangle=s(s+1)
$$


for any state $\psi$, we can now rewrite Eq. (10) as follows

$$
\nu(\psi)=1-\frac{\bar{S}_{\psi}^{2}}{s^{2}}
$$

where $\bar{S}_{\psi}^{2} \equiv \sum_{j}\left\langle\psi\left|S_{j}\right| \psi\right\rangle^{2}$ is the squared length of the mean-spin vector.

Entanglement in spin systems - According to the modern theory of quantum entanglement (see [6] and references therein), the completely entangled (CE) states of a given system are defined to be the states with the maximal amount of total uncertainty calculated for the set of basic observables. In the case of a single spin, the basic observables are given by the operators $S_{x}, S_{y}, S_{z}$, so that

$$
\mathbb{V}\left(\psi_{C E}\right)=\max \mathbb{V}(\psi)=s(s+1),
$$

where maximum is taken over all states in the $(2 s+1)$-dimensional Hilbert space of spin states. This definition assumes the following condition

$$
\left\langle\psi_{C E}\left|S_{j}\right| \psi_{C E}\right\rangle=0 \quad \forall j=x, y, z,
$$

that can be used as the operational definition of complete entanglement [9] (also see discussion in Appendix A). All other entangled states can be obtained from the completely entangled states by means of SLOCC (stochastic local operations assisted by classical communications) [10].

Thus, by definition, maximally squeezed spin states coincide with the completely entangled spin states. Moreover, it has been shown in Ref. [11] that the square root of the measure of squeezing (11) gives the measure of the amount of entanglement carried by a pure spin state:

$$
\mu(\psi) \sqrt{\nu(\psi)}=\sqrt{1-\frac{\bar{S}_{\psi}^{2}}{s^{2}}} .
$$

Let us now note that an organic connection between the notions of entanglement and squeezing is under discussion for some time $[12,13]$. In the most of works, a kind of "two-mode" entanglement [14] was considered for the two-qubit (two spin-1/2) systems. In the papers 


\section{Barıș Öztop, Alexander A. Klyachko and Alexander S. Shumovsky}

$[15,17,16]$, the possibility of entanglement of a single spin-1 object has been shown. It should be stressed that our present consideration shows equivalence of the spin squeezing and spin entanglement for a single object with any $s=1$. At $s>1$, squeezing implies entanglement but not vice versa.

Squeezed states of spin-1 - As an illustrative physical example of some considerable interest, examine now squeezing of a single spin-1 object. Stress that hereafter by "spin" we mean an object with the dynamic symmetry given by the $\mathrm{SU}(2)$ group, so that it can be a real spin, isospin, angular momentum, etc.

A general pure state in the three-dimensional Hilbert $\mathcal{H}_{3}$ space has the form

$$
|\psi\rangle=\sum_{s=-1}^{1} \psi_{s}|s\rangle, \quad \sum_{s=-1}^{1}\left|\psi_{s}\right|^{2}=1 .
$$

In the basis $|s\rangle$, spin operators have the following representation

$$
\begin{aligned}
& S_{x}=(|+1\rangle+|-1\rangle)\langle 0|+| 0\rangle(\langle+1|+\langle-1|), \\
& S_{y}=-i(|+1\rangle-|-1\rangle)\langle 0|+i| 0\rangle(\langle+1|-\langle-1|), \\
& S_{z}=|+1\rangle\langle+1|-|-1\rangle\langle-1| .
\end{aligned}
$$

Since the spin-displacement operator in Eq. (3) has the form

$$
\left(\begin{array}{ccc}
\frac{1}{2}[1+\cos (|\alpha| \sqrt{2})] & \frac{e^{i \phi}}{\sqrt{2}} \sin (|\alpha| \sqrt{2}) & \frac{e^{2 i \phi}}{2}[1-\cos (|\alpha| \sqrt{2})] \\
-\frac{e^{-i \phi}}{\sqrt{2}} \sin (|\alpha| \sqrt{2}) & \cos (|\alpha| \sqrt{2}) & \frac{e^{i \phi}}{\sqrt{2}} \sin (|\alpha| \sqrt{2}) \\
\frac{e^{-2 i \phi}}{2}[1-\cos (|\alpha| \sqrt{2})] & -\frac{e^{-i \phi}}{\sqrt{2}} \sin (|\alpha| \sqrt{2}) & \frac{1}{2}[1+\cos (|\alpha| \sqrt{2})]
\end{array}\right)
$$

the coherent states for spin 1 have the following general form

$$
\begin{aligned}
|\alpha\rangle & =\frac{e^{2 i \phi}}{2}[1-\cos (|\alpha| \sqrt{2})]|+1\rangle+\frac{e^{i \phi}}{\sqrt{2}} \sin (|\alpha| \sqrt{2})|0\rangle \\
& +\frac{1}{2}[1+\cos (|\alpha| \sqrt{2})]|-1\rangle .
\end{aligned}
$$

Here $\phi=\arg \alpha$. It is easily seen that the measure of squeezing (11) as well as the measure of entanglement (13) have zero value for the state (16) for all $\alpha \in \mathbb{C}$. 
To determine the maximally squeezed (completely entangled) states of spin-1 system under consideration let us use the conditions (12), which for the state (14) take the form

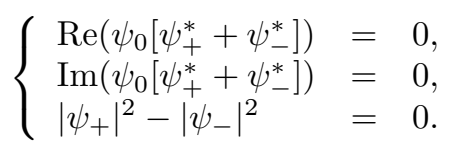

In addition, one should take into account the normalization condition in (14).

Setting aside the phase of the state (14), we arrive at the conclusion that the state (14) is specified by five real parameters that, in the case of completely entangled states, obey the four equations (three equations (17) plus normalization condition in (14). Thus, there are infinitely many completely entangled and hence maximally squeezed states of a single spin-1 system. In particular, the states

$$
|0\rangle, \quad \frac{1}{\sqrt{2}}(|+1\rangle \pm|-1\rangle)
$$

form the orthogonal basis of maximally squeezed states in the threedimensional Hilbert space $\mathcal{H}_{3}$ of spin- 1 states. In fact, the complement of the subspace $\mathcal{H}_{3}^{(c o h)} \subset \mathcal{H}_{3}$, formed by the coherent states (16), with respect to the whole space $\mathcal{H}_{3}$ corresponds to the squeezed (entangled) states of spin 1. All those states can be obtained from the basis (18) by means of SLOCC (in other words, by action of operators from the complexified dynamic symmetry group [10]).

Entanglement of a single spin-1 and nonlocality - We have shown that the notions of spin-squeezing and spin-entanglement are equivalent, and that the spin-entangled states can exist for a single spin. This result conflicts with the common opinion that entanglement always assumes nonlocality of the system (e.g., see discussions in Refs. $[6,18])$. The nonlocality simply means that measurements on spatially separated parts of a quantum system may instantaneously influence one another $[19,20]$. Physically this is caused by quantum correlations between the parts of the system [20]. Once created, those correlations exist even after spatial separation of parts. In many important cases, creation of entanglement assumes initial locality of the system (for example, decay of an excited atomic state with creation of 


\section{Barıș Öztop, Alexander A. Klyachko and Alexander S. Shumovsky}

polarization-entangled photon twins). Thus, despite of the fact that nonlocality is one of the most important distinguishing features of quantum mechanics with respect to always "local" classical mechanics, we should conclude that it cannot be considered as a necessarily accompaniment of entanglement.

In particular, this fact follows from the formal equivalence of the single spin- 1 entanglement and that of two spin- $1 / 2$ "particles" considered in the symmetric subspace of the four-dimensional Hilbert space $\mathcal{H}_{2} \otimes \mathcal{H}_{2}$ (see discussion in Ref. [16]). This comes directly from the Clebsch-Gordon decomposition of irreducible representations of two $\mathrm{SU}(2)$ into irreducible subspaces [21]:

$$
\mathcal{H}_{2} \otimes \mathcal{H}_{2}=\mathcal{H}_{3} \oplus \mathcal{H}_{A}
$$

Here $\mathcal{H}_{2}$ is the two-dimensional space spanned by the base vectors $|\uparrow\rangle$ and $|\downarrow\rangle$, the three-dimensional space $\mathcal{H}_{3}$ is spanned by the symmetric vectors

$$
\begin{aligned}
|+1\rangle & \sim|\uparrow \uparrow\rangle, \\
|0\rangle & \sim \frac{1}{\sqrt{2}}(|\uparrow \downarrow\rangle+|\downarrow \uparrow\rangle), \\
|-1\rangle & \sim|\downarrow \downarrow\rangle,
\end{aligned}
$$

and $\mathcal{H}_{A}$ corresponds to the antisymmetric vector

$$
|A\rangle \sim \frac{1}{\sqrt{2}}(|\uparrow \downarrow\rangle-|\downarrow \uparrow\rangle) .
$$

Thus, a single spin-1 object can be associated with two qubits defined in the symmetric subspace of the four-dimensional Hilbert space. In the case of spin-1 particle (local object), the two qubits correspond to the intrinsic degrees of freedom.

Physical examples - It follows from the above results that any system with the dynamic symmetry $\mathrm{SU}(2)$ and dimension of the Hilbert space $d \geq 3$ may manifest squeezing and hence entanglement independent of the physical nature of this system. For example, it can be isotriplet of $\pi$-mesons, in which the maximally squeezed state can be associated with the neutral $\pi^{0}$ meson [6]. It can be the spin-1 atoms like ${ }^{87} \mathrm{Rb}$ and ${ }^{23} \mathrm{Na}$, widely used in investigation of Bose-Einstein condensation, and even more exotic systems like vector 
Squeezed and entangled states of a single spin

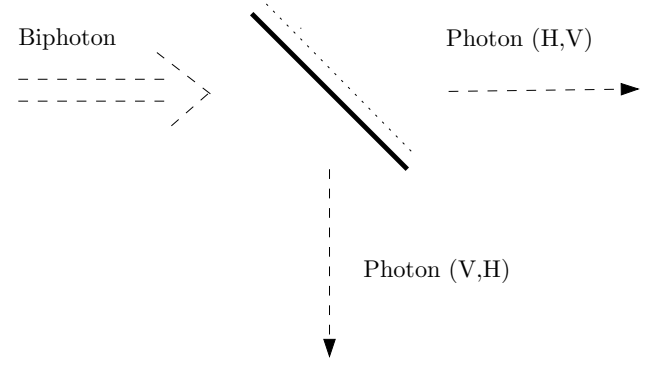

Figure 1: Splitting of unpolarized biphoton into photons with different linear polarization (either Horizontal or Vertical).

mesons and three spin-1 gauge bosons in the standard model [22], in which spin-1 entanglement (squeezing) may also be realized.

A less exotic example is provided by a biphoton - photon twins created at once and propagating in the same direction (concerning biphotons, see [23]). Because of the Bosonic nature, the antisymmetric state is forbidden for the biphoton [24], so that it exists in the three-dimensional space spanned by the symmetric vectors (19) (here arrows correspond to the orthogonal polarizations, vertical and horizontal). Unlike a single photon, biphoton can be considered as a "particle" with three polarizations, so that it represents a spin-1 object. Through the use of a beam splitter, biphoton can "decay" into two photons (see Fig. 1). In the case of maximally squeezed initial biphoton state $|0\rangle$, the two photons after decay form a two-qubit entangled system. One of the qubits is given by the polarization of photons, while another "geometrical" qubit corresponds to the possible paths of photons after beam splitter.

Another example of interest is given by a three-level atom. Consider first the so-called $\lambda$-configuration of dipole transitions (Fig. 2), corresponding to the hyperfine levels of cesium (Cs). Namely, the excited state corresponds to the sublevel $\left(6 S_{1 / 2}, F=3, m=0\right)$, while the ground states are the sublevels $\left(6 P_{1 / 2}, F=3, m= \pm 1\right)$. Transition from the excited state to the state with $m=-1(m=+1)$ gives rise to the right (left) circularly polarized photons of the same wavelength $(852.36 \mathrm{~nm})$ [25]. Transitions of this type are usually used 
Barıș Öztop, Alexander A. Klyachko and Alexander S. Shumovsky

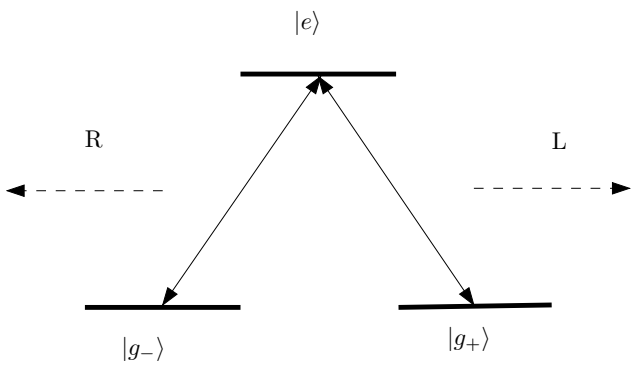

Figure 2: $\lambda$-type transition in Cs atom.

for creation of the atom-photon entanglement [26]. The atom-photon interaction in a cavity is described by the Hamiltonian

$$
H_{\text {int }}=g\left(R_{e g_{+}} a_{L}+R_{e g_{-}} a_{R}+H . c .\right) .
$$

Here $g$ is the coupling constant, $R_{j k}=|j\rangle\langle k|$ is the atomic transition operator and $a_{P}$ is the annihilation operator describing a photon with polarization $P=R, L$.

Let us now associate the atomic levels with the states of "spin-1" as follows:

$$
|e\rangle=|0\rangle, \quad\left|g_{ \pm}\right\rangle=| \pm 1\rangle .
$$

Then, it follows from the above discussion that the excited atomic state $|e\rangle$ is maximally squeezed and hence completely entangled with respect to the measurement of "spin" operators

$$
\begin{aligned}
S_{x} & =\frac{1}{\sqrt{2}}\left(R_{g_{+} e}+R_{g_{-} e}+H . c .\right) \\
S_{y} & =\frac{-i}{\sqrt{2}}\left(R_{g_{+} e}-R_{g_{-} e}-H . c .\right) \\
S_{Z} & =R_{g_{+} g_{+}}-R_{g_{-} g_{-}} .
\end{aligned}
$$

Their expectation values in the state $|e\rangle$ are equal to zero, which corresponds to the condition (12).

Assume that the system is initially prepared in the state

$$
\left|\psi_{1}\right\rangle=|e\rangle \otimes|\mathrm{vac}\rangle
$$


(atom is in the excited state and cavity field is in the vacuum state). It is then clear that the radiative decay of the state $\left|\psi_{1}\right\rangle$ gives rise to the state

$$
\left|\psi_{2}\right\rangle=\frac{1}{\sqrt{2}}\left(\left|g_{+}\right\rangle \otimes\left|1_{L}\right\rangle+\left|g_{-}\right\rangle \otimes\left|1_{R}\right\rangle\right),
$$

which is the atom-field two-qubit entangled state. Namely, the atomic qubit of states $\left|g_{ \pm}\right\rangle$is entangled with the photon polarization qubit. The atomic qubit can be specified by the three observables (an analogue of Pauli operators for spin $1 / 2$ )

$$
\begin{aligned}
\sigma_{x} & =\left(R_{g_{+} g_{-}}+R_{g_{-} g_{+}}\right), \\
\sigma_{y} & =-i\left(R_{g_{+} g_{-}}-R_{g_{-} g_{+}}\right), \\
\sigma_{z} & =\left(R_{g_{+} g_{+}}-R_{g_{-} g_{-}}\right) .
\end{aligned}
$$

In turn, the photon polarization qubit is specified by the Stokes operators

$$
\begin{aligned}
\mathcal{S}_{x} & =a_{R}^{+} a_{L}+a_{L}^{+} a_{R}, \\
\mathcal{S}_{y} & =-i a_{R}^{+} a_{L}+i a_{L}^{+} a_{R}, \\
\mathcal{S}_{z} & =a_{R}^{+} a_{R}-a_{L}^{+} a_{L} .
\end{aligned}
$$

It can be easily seen that in the case of a lossless cavity the timedependent probability to observe the system in the state $\left|\psi_{1}\right\rangle$ is

$$
P_{1}(t)=\cos ^{2} \Omega t+\frac{\Delta^{2}}{4 \Omega^{2}} \sin ^{2} \Omega t,
$$

where $\Omega=\frac{1}{2} \sqrt{8 g^{2}+\Delta^{2}}$ and $\Delta$ denotes detuning between the atomic transition and cavity mode. The maximal value of $P_{1}^{(\max )}=1$ is achieved at $t=k \pi(k=0,1, \cdots)$, while the minimal value

$$
P_{1}^{(m i n)}=\frac{\Delta^{2}}{8 g^{2}+\Delta^{2}}
$$

vanishes at $\Delta \rightarrow 0$. In turn, the probability to have atom-field entanglement described by the state $\left|\psi_{2}\right\rangle$ is

$$
P_{2}(t)=1-P_{1}(t)=\frac{8 g^{2}}{8 g^{2}+\Delta^{2}} \sin ^{2} \Omega t .
$$


Barıș Öztop, Alexander A. Klyachko and Alexander S. Shumovsky

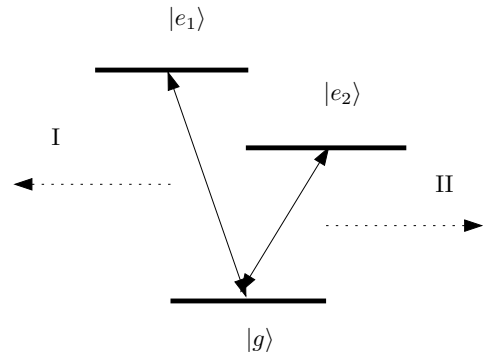

Figure 3: $V$-type atomic transition.

Similar results can be obtained for the two other three-level configurations. For example, in the case of three-level atom with $V$-type transitions (Fig. 3), the spin-1 states can be associated with the atomic states as follows

$$
|+1\rangle=\left|e_{1}\right\rangle, \quad|0\rangle=|g\rangle, \quad|-1\rangle=\left|e_{2}\right\rangle .
$$

In this case, initial state $|g\rangle \otimes\left|1_{1}, 1_{2}\right\rangle$ is again entangled with respect to spin- 1 operators. It is transformed into the atom-field entangled state $\left(g_{1}\left|e_{1}\right\rangle \otimes\left|1_{2}\right\rangle+g_{2}\left|e_{2}\right\rangle \otimes\left|1_{1}\right\rangle\right)$, where $g_{j}$ denotes the coupling constant for the corresponding allowed transition.

In the case of the so-called ladder configuration (Fig. 4), the intermediate state $|i\rangle$ can be associated with the squeezed states $|0\rangle$, while $|e\rangle=|+1\rangle$ and $|g\rangle=|-1\rangle$. Evolution from the initial state $|i\rangle \otimes\left|1_{2}\right\rangle$ creates the atom-field entangled state

$$
g_{1}|g\rangle \otimes\left|1_{1}, 1_{2}\right\rangle+g_{2}|e\rangle \otimes\left|0_{1}, 0_{2}\right\rangle .
$$

\section{Conclusion}

We have shown complete equivalence between the notions of squeezing and entanglement in spin systems - in general, in systems with the dynamic symmetry given by the $\mathrm{SU}(2)$ group. In turn, spin-coherent states are always unentangled.

The spin squeezing and spin entanglement can be measured in the same way by means of the length of mean-spin vector (see Eqs. (11) 
Squeezed and entangled states of a single spin

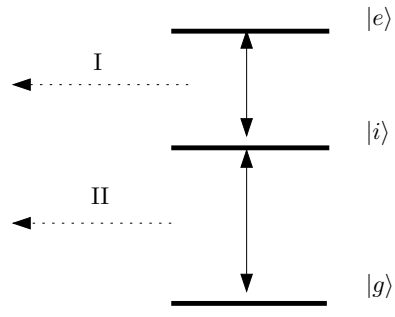

Figure 4: Ladder-type atomic transition.

and (13)). This shows that the amount of either squeezing or entanglement carried by a given state of a spin-system can be obtained by only three measurement of the components of spin operator.

In the case of spin $s \geq 1$, both spin squeezing and spin entanglement can be observed for a single local object (particle). For example, three-level atoms can be prepared in squeezed states. This shows that association of entanglement with quantum nonlocality, which is widely used for specification of entanglement since the pioneering paper by Einstein, Podolsky and Rosen [19], is not the necessary condition of entanglement.

The nonlocality of entanglement simply means that measurements on spatially separated parts of a quantum system may instantaneously influence one another. Physically this is caused by the quantum correlations between the parts of the system[20]. Once created, those correlations keep on existence even after the spatial separation of parts.

On one hand, the nonlocality is probably the main distinguishing feature of quantum mechanics regarding classical physics. On the other hand, this notion does not contain any quantification of distance between separated entangled parts of a quantum system. Thus, it seems to be natural to assume that a quantum system with strongly correlated intrinsic parts may manifest entanglement as a local object even without spatial separation of parts [6]. We have shown that in some processes, this intrinsic spin-entanglement can be transformed into entanglement of two separated qubits. Namely, spin-squeezed three-level atom gives rise to entangled state of photon and atom 
qubits.

Our consideration has shown ubiquity of the notion of entanglement as a manifestation of quantum fluctuations of observables at their extreme.

\section{Acknowledgments}

One of the authors (B. Ö.) would like to acknowledge the Scientific and Technical Research Council of Turkey (TÜBİTAK) for financial support.

\section{Appendix A: Entanglement and observables}

In the description of quantum entanglement, the analysis of the dynamic symmetry of a given physical system plays a determinative role $[12,6]$.

Let us now note that an idealized von Neumann approach to quantum mechanics, based on assumption that all Hermitian operators represent measurable quantities, was first put into question by Wick, Wightman and Wigner [27] in 1952.

"It is quite true that the assumption that all Hermitian operators represent measurable quantities is often represented as integral part of the general scheme of quantum mechanics. It is also true that in the case of the ordinary non-relativistic quantum mechanics this assumption, implausible as it sounds for all but very simplest operators, is not subject to any very serious objection. ... It is indeed surprising that the nature of such scheme has not been more widely discussed."

Later Robert Hermann [28] argued soundly that the basic principles of quantum mechanics require that measurable observables should form a Lie algebra $\mathcal{L}$ of (skew)Hermitian operators acting in Hilbert space $\mathcal{H}$ of the quantum system in question. We refer to $\mathcal{L}$ as Lie algebra of observables and to the corresponding Lie group $G=\exp (i \mathcal{L})$ as dynamical symmetry group of the quantum system.

Restrictions on available observations are of fundamental importance for physics in general, and for quantum information specifically. 
The latter case usually deals with correlated states of a quantum system with macroscopically separated spatial components, where only local measurements are feasible. For example, the dynamical group of bipartite system $\mathcal{H}=\mathcal{H}_{A} \otimes \mathcal{H}_{B}$ with full access to local degrees of freedom amounts to $\mathrm{SU}\left(\mathcal{H}_{A}\right) \times \mathrm{SU}\left(\mathcal{H}_{B}\right)$. Without such restrictions, the dynamical group $G=\mathrm{SU}(\mathcal{H})$ would act transitively on pure states $\psi \in \mathcal{H}$, which makes them all equivalent. In this case there would be no place for entanglement and other subtle quantum phenomena based on intrinsic differences between quantum states.

Possible ambiguous choice of the dynamic symmetry of a physical system causes a certain relativity of entanglement. For example, in quantum information qutrit is defined to be a state in threedimensional Hilbert space $\mathcal{H}_{3}$. Thus, the general dynamic symmetry associated with qutrits is $\mathrm{SU}\left(\mathcal{H}_{3}\right)=\mathrm{SU}(3)$. This assumes eight independent basic observables (Gell-Mann matrices) specifying a given state of qutrit [29]. At the same time, three-state system can also be associated with the lower dynamic symmetry $\mathrm{SU}(2) \subset \mathrm{SU}(3)$, corresponding to spin- 1 systems. The reduced dynamic symmetry assumes only three-basic observables. It is clear a priori that the $\mathrm{SU}(2)$ and $\mathrm{SU}(3)$ qutrits have different properties of entangled states. Namely, single qutrit that, as we have shown, can manifest spin-entanglement, can never be entangled in the $\mathrm{SU}(3)$ domain.

\section{Appendix B: Proof of universality of measure (13)}

Following Ref. [30], let us now demonstrate universality of the measure of entanglement (13) for pure states.

Let $\operatorname{Herm}(\mathcal{H})$ be space of all Hermitian operators acting in Hilbert space $\mathcal{H}$ with trace metric $\operatorname{Tr}_{\mathcal{H}}(X Y)$. For simple algebra $\mathcal{L}$ restriction of the trace metric onto $\mathcal{L}$ is proportional to the Cartan-Killing form

$$
\operatorname{Tr}_{\mathcal{H}}(X Y)=D_{\mathcal{H}} \cdot(X, Y)_{K}, \quad X, Y \in \mathcal{L}
$$

with the coefficient $D_{\mathcal{H}}$ known as Dynkin index [31]. Consider now orthogonal projection $\rho_{\mathcal{L}}$ of $\rho:=|\psi\rangle\langle\psi| \in \operatorname{Herm}(\mathcal{H})$ into subalgebra $\mathcal{L} \subset \operatorname{Herm}(\mathcal{H})$, so that $\operatorname{Tr}_{\mathcal{H}}(\rho X)=\operatorname{Tr}_{\mathcal{H}}\left(\rho_{\mathcal{L}} X\right), \forall X \in \mathcal{L}$. The 


\section{Barıș Öztop, Alexander A. Klyachko and Alexander S. Shumovsky}

projection $\rho_{\mathcal{L}}$ is closely related to the average of basic observable

$$
\begin{aligned}
X_{\psi} & =\sum_{\alpha} \operatorname{Tr}_{\mathcal{H}}\left(\rho X_{\alpha}\right) X_{\alpha}=\sum_{\alpha} \operatorname{Tr}_{\mathcal{H}}\left(\rho_{\mathcal{L}} X_{\alpha}\right) X_{\alpha} \\
& =D_{\mathcal{H}} \sum_{\alpha}\left(\rho_{\mathcal{L}}, X_{\alpha}\right)_{K} X_{\alpha}=D_{\mathcal{H}} \cdot \rho_{\mathcal{L}} .
\end{aligned}
$$

Therefore

$$
\left\langle\psi\left|X_{\psi}\right| \psi\right\rangle=\operatorname{Tr}_{\mathcal{H}}\left(\rho X_{\psi}\right)=\operatorname{Tr}_{\mathcal{H}}\left(\rho_{\mathcal{L}} X_{\psi}\right) D_{\mathcal{H}} \operatorname{Tr}_{\mathcal{H}}\left(\rho_{\mathcal{L}}^{2}\right)
$$

and the total variance (6) can be written in the form

$$
\mathbb{V}(\psi)=C_{\mathcal{H}}-\left\langle\psi\left|X_{\psi}\right| \psi\right\rangle C_{\mathcal{H}}-D_{\mathcal{H}} \cdot \operatorname{Tr}_{\mathcal{H}}\left(\rho_{\mathcal{L}}^{2}\right) .
$$

For simple algebra the Casimir $C_{\mathcal{H}}$ and Dynkin index $D_{\mathcal{H}}$ are given by equations

$$
C_{\mathcal{H}}=(\lambda, \lambda+2 \delta), \quad D_{\mathcal{H}} \frac{\operatorname{dim} \mathcal{H}}{\operatorname{dim} \mathcal{L}}(\lambda, \lambda+2 \delta),
$$

where $\lambda$ denotes the highest weight of irreducible representation $\mathcal{H}$ and $2 \delta$ is the sum of positive roots of $\mathcal{L}$. For example, for full algebra of traceless Hermitian operators $\mathcal{L}=\mathfrak{s u}(\mathcal{H})$ we have

$$
C_{\mathcal{H}}=\operatorname{dim} \mathcal{H}-\frac{1}{\operatorname{dim} \mathcal{H}}, \quad D_{\mathcal{H}}=1 .
$$

In general, algebra $\mathcal{L}$ splits into simple components $\mathcal{L}=\bigoplus_{A} \mathcal{L}_{A}$ and its irreducible representation $\mathcal{H}$ into tensor product $\mathcal{H}=\bigotimes_{A} \mathcal{H}_{A}$. In this case equation (24) should be modified as follows

$$
\mathbb{V}(\psi)=\sum_{A}\left[C_{\mathcal{H}_{A}}-D_{\mathcal{H}_{A}} \cdot \operatorname{Tr}_{\mathcal{H}_{A}}\left(\nu_{A}^{2} \rho_{\mathcal{L}_{A}}^{2}\right)\right],
$$

where $\nu_{A}=\operatorname{dim} \mathcal{H} / \operatorname{dim} \mathcal{H}_{A}$.

In quantum information setting $\mathcal{L}_{A}$ is the full algebra of traceless Hermitian operators $X_{A}: \mathcal{H}_{A} \rightarrow \mathcal{H}_{A}$. In this case everything can be done explicitly. By definition of reduced states $\rho_{A}$ we have

$$
\operatorname{Tr}_{\mathcal{H}}\left(\rho X_{A}\right) \operatorname{Tr}_{\mathcal{H}_{A}}\left(\rho_{A} X_{A}\right)=\nu_{A}^{-1} \operatorname{Tr}_{\mathcal{H}}\left(\rho_{A} X_{A}\right) .
$$


Comparing this with equation $\operatorname{Tr}_{\mathcal{H}}\left(\rho X_{A}\right)=\operatorname{Tr}_{\mathcal{H}}\left(\rho_{\mathcal{L}_{A}} X_{A}\right), \forall X_{A} \in \mathcal{L}_{A}$ characterizing the projection $\rho_{\mathcal{L}_{A}} \in \mathcal{L}_{A}$ we infer

$$
\rho_{\mathcal{L}_{A}}=\nu_{A}^{-1} \rho_{A}^{0},
$$

where $\rho_{A}^{0}=\rho_{A}-\frac{1}{\operatorname{dim} \mathcal{H}_{A}} \mathbf{I}$ is traceless part of $\rho_{A}$. This allows to calculate the trace

$$
\operatorname{Tr}_{\mathcal{H}_{A}}\left(\rho_{\mathcal{L}_{A}}^{2}\right) \nu^{-2}\left[\operatorname{Tr}_{\mathcal{H}_{A}}\left(\rho_{A}^{2}\right)-\frac{1}{\operatorname{dim} \mathcal{H}_{A}}\right]
$$

Plugging this into equation (27) and using (26) we finally get

$$
\mathbb{V}(\psi)=\sum_{A}\left[\operatorname{dim} \mathcal{H}_{A}-\operatorname{Tr}_{\mathcal{H}_{A}}\left(\rho_{A}^{2}\right)\right] .
$$

As an example, consider completely entangled state $\psi$ for which $\rho_{A}=$ $\frac{1}{\operatorname{dim} \mathcal{H}_{A}} \mathbf{I}$. This gives the maximum of the total variance

$$
\mathbb{V}_{\text {max }}=\mathbb{V}_{\text {ent }}=\sum_{A}\left(\operatorname{dim} \mathcal{H}_{A}-\frac{1}{\operatorname{dim} \mathcal{H}_{A}}\right) .
$$

The minimum of the total variance is attained for coherent (=separable) state $\psi$, for which reduced states $\rho_{A}$ are pure. Hence

$$
\mathbb{V}_{\text {min }}=\mathbb{V}_{\text {coh }}=\sum_{A}\left(\operatorname{dim} \mathcal{H}_{A}-1\right)
$$

Combining these equations we can write down our measure of entanglement (13) explicitly for a multicomponent system $\mathcal{H}=\bigotimes_{A} \mathcal{H}_{A}$ of arbitrary format

$$
\mu(\psi)^{2}=\frac{\sum_{A}\left[1-\operatorname{Tr}\left(\rho_{A}^{2}\right)\right]}{\sum_{A}\left(1-\frac{1}{\operatorname{dim} \mathcal{H}_{A}}\right)} .
$$

\section{References}

[1] D. Stoler, Phys. Rev. D 1, 3217 (1970); ibidem 4, 1925 (1971).

[2] N.N. Bogoliubov, J. Phys. (USSR) 11, 23 (1947). 
Barıș Öztop, Alexander A. Klyachko and Alexander S. Shumovsky

[3] J.M. Radcliffe, J. Phys. A: Math. General 4, 313 (1971): F.T. Arecchi, E. Courtens, R. Gilmore, and H. Thomas, Phys. Rev. A 6, 2211 (1972).

[4] R.J. Glauber, Phys. Rev. Lett. 10, 84 (1963); Phys. Rev. 130, 2529 (1963).

[5] A.O. Barut and L. Girardello, Commun. Math. Phys. 21, 41 (1971); R. Gilmore, Lie groups, Lie algebras, and some of their applications (Wiley, New York, 1974); I.A. Malkin and V.I. Man'ko, Dynamic symmetries and cohrent states of quantum systems (Nauka, Moscow, 1979); A.M. Perelomov, Generalized coherent states and their applications (Springer-Verlag, Berlin, 1986).

[6] A.A. Klyachko and A.S. Shumovsky, J. Phys: Conf. Series 36, 87 (2006).

[7] V.V. Dodonov, J. Opt. B 4, R1 (2002).

[8] M. Kitagawa and M. Ueda, Phys. Rev. A 47, 5138 (1993).

[9] M.A. Can, A.A. Klyachko, and A.S. Shumovsky, Phys. Rev. A 66, 022111 (2002).

[10] W. Dür, G. Vidal, and J.I. Cirac, Phys. Rev. A 62, 062314 (2000); F. Verstraete, J. Dehaene, and B. De Moor, Phys. Rev. A 68, 012103 (2003).

[11] A.A. Klyachko, B. Öztop and A.S. Shumovsky, Appl. Phys. Lett. 88, $124102(2006)$.

[12] A.A. Klyachko, E-print quant-ph/0206012.

[13] J. Hald, J.L. Sørensen, C. Schori, and E.S. Polzik, Phys. Rev. A 83, 1319 (1999); A. Sørensen, L.M. Duan, J.I. Cirac, and P. Zoller, Nature (London) 409, 63 (2001); U.V. Poulsen and K. Mølmer, Phys. Rev. A 64, 013616 (2001); A. Sørensen and K. Mølmer, Phys. Rev. Lett. 86, 4431 (2001); L.K. Thomsen, S. Mancini, and H.M. Wiseman, Phys. Rev. A 65, 061801 (2002); J.K. Stokton, J.M. Geremia, A.C. Doherty, and H. Mabuchi, Phys. Rev. A 67, 022112 (2003); D.W. Berry and B.C. Sanders, J. Phys. A 38, L205 (2005). 
Squeezed and entangled states of a single spin

[14] R. Loudon and P.L. Knight, J. Mod. Opt. 34, 709 (1987).

[15] A.A. Klyachko and A.S. Shumovsky, J. Opt. B: Quant. and Semiclas. Optics 5, S322 (2003).

[16] M.A. Can, A.A. Klyachko, and A.S. Shumovsky, J. Opt. B: Quant. Semiclass. Opt. 7, L1 (2005).

[17] H. Barnum, E. Knill, G. Ortiz, R. Somma, and L. Viola, Phys. Rev. Lett. 92, 107902 (2004).

[18] S.J. van Enk, Phys. Rev. A 67, 022303 (2003); Phys. Rev. A 72, 064306 (2005).

[19] A. Einstein, B. Podolsky, and N. Rosen, Phys. Rev. 47, 777 (1935).

[20] J.S. Bell, Rev. Mod. Phys. 38, 447 (1966).

[21] M. Hamermesh, Group theory and its applications to physical problems (Addison-Wesly, Reding Mass., 1962).

[22] N. Cottingham and D.A. Greenwood, An Introduction to Standard Model of Elementary Particle Physics (Cambridge University Press, New York, 1998).

[23] A.V. Burlakov, M.V. Chechova, O.A. Karabutova, D.N. Klyshko, and S.P. Kulik, Phys. Rev. A 60, R4209 (1999); M.B. Nasr, A.F. Abouraddy, M.C. Booth, B.E.A. Saleh, A.V. Sergienko, M.C. Teich, U. Kempe, amd R. Wollescheensky, Phys. Rev. A 65, 023816 (2002); G. DiGiuseppe, M. Atature, M. Shaw, A.V. Sergienko, B.E.A. Saleh, M.C. Teich, A.J. Miller, S.W. Nam, and J.M. Martinis, Phys. Rev. A 68, 063817 (2003); Y.I. Bogdanov, M.V. Chechova, S.P. Kulik, G.A. Maslennikov, A.A. Zhukov, C.H. Oh, and M.K. Tey Phys. Rev. Lett. 93, 230503 (2004); M.V. Chechova, L.A. Krivitsky, S.P. Kulik, and G.A. Maslennikov, Phys. Rev. A 70, 053801 (2004).

[24] V.B. Berestetskii, E.M. Lifshitz, and L.P. Pitaevskii, 1982 Quantum Electrodynamics (Pergamon Press, Oxford, 1982).

[25] W. Lange and H.J. Kimble, Phys. Rev. A 61, 063817 (2000). 
Barıș Öztop, Alexander A. Klyachko and Alexander S. Shumovsky

[26] B.B. Blinov, D.L. Moehring, L.-M. Duan, and C. Monroe, Nature 428, 153 (2004).

[27] G.C. Wick, A.S. Wightman and E.P. Wigner, Phys. Rev. 88, 101 (1952).

[28] R. Hermann , Lie groups for physicists (Benjamin, New York, 1966).

[29] C.M. Caves and G.J. Milburn, Opt. Commun. 179, 439 (2000).

[30] A.A. Klyachko, B. Öztop and A.S. Shumovsky, Phys. Rev. A (2007), in print; quant-ph/0606119.

[31] A.L. Onishchik and E.B. Vinberg (editors), Lie groups and Lie algebras III, Encyclopedia of Math. Science Vol. 41 (Springer, Berlin, 1994). 